\title{
DNA-based identification of novel bovine casein gene variants
}

\author{
J. L. Gallinat, ${ }^{*}$ S. Qanbari,† C. Drögemüller,ł E. C. G. Pimentel,†§ G. Thaller, ${ }^{*}$ and J. Tetens* ${ }^{* 1}$ \\ *Institute of Animal Breeding and Husbandry, Christian-Albrechts-University Kiel, D-24118 Kiel, Germany \\ †Institute of Animal Breeding and Genetics, University of Göttingen, D-37075 Göttingen, Germany \\ ‡Institute of Genetics, Vetsuisse Faculty, University of Bern, CH-3001 Bern, Switzerland \\ $\S$ Department of Animal Breeding, Faculty of Organic Agricultural Sciences, University of Kassel, D-37213 Witzenhausen, Germany
}

\section{ABSTRACT}

In cattle, at least 39 variants of the 4 casein proteins $\left(\alpha_{\mathrm{S1}^{-}}, \beta-, \alpha_{\mathrm{S} 2^{-}}\right.$and $\kappa$-casein $)$ have been described to date. Many of these variants are known to affect milkproduction traits, cheese-processing properties, and the nutritive value of milk. They also provide valuable information for phylogenetic studies. So far, the majority of studies exploring the genetic variability of bovine caseins considered European taurine cattle breeds and were carried out at the protein level by electrophoretic techniques. This only allows the identification of variants that, due to amino acid exchanges, differ in their electric charge, molecular weight, or isoelectric point. In this study, the open reading frames of the casein genes CSN1S1, CSN2, CSN1S2, and CSN3 of 356 animals belonging to 14 taurine and 3 indicine cattle breeds were sequenced. With this approach, we identified 23 alleles, including 5 new DNA sequence variants, with a predicted effect on the protein sequence. The new variants were only found in indicine breeds and in one local Iranian breed, which has been phenotypically classified as a taurine breed. A multidimensional scaling approach based on available SNP chip data, however, revealed an admixture of taurine and indicine populations in this breed as well as in the local Iranian breed Golpayegani. Specific indicine casein alleles were also identified in a few European taurine breeds, indicating the introgression of indicine breeds into these populations. This study shows the existence of substantial undiscovered genetic variability of bovine casein loci, especially in indicine cattle breeds. The identification of new variants is a valuable tool for phylogenetic studies and investigations into the evolution of the milk protein genes.

Key words: casein, genetic variant, Bos indicus, Bos taurus

Received July 5, 2012.

Accepted September 13, 2012.

${ }^{1}$ Corresponding author: jtetens@tierzucht.uni-kiel.de

\section{INTRODUCTION}

The 4 caseins $\left(\alpha_{\mathrm{S1}^{-}}, \alpha_{\mathrm{S2}^{-}}, \beta\right.$ - and $\left.\kappa-\mathrm{CN}\right)$ account for more than $75 \%$ of the whole bovine milk protein. They are encoded by 4 genes mapped to BTA 6 (Hayes and Petit, 1993) in a tightly linked $250-\mathrm{kb}$ cluster. The gene order is CSN1S1 ( $\alpha_{\mathrm{S} 1}-\mathrm{CN}$-encoding gene), CSN2 ( $\beta-\mathrm{CN}$ encoding gene), CSN1S2 ( $\alpha_{\mathrm{S}_{2}} \mathrm{CN}-\mathrm{Cncoding}$ gene), and CSN3 ( -CN-encoding gene; Threadgill and Womack, 1990). Caseins do not only provide the suckling infant with calcium, phosphate, and amino acids, but also have an influence on milk-production traits and cheesemaking properties (Boettcher et al., 2004; Wedholm et al., 2006; Nilsen et al., 2009). Consequently, caseins have already been investigated intensively at the protein as well as at the DNA level, especially in cattle. All bovine casein genes have been shown to be polymorphic (Farrell et al., 2004). To date, 9 protein variants of $\alpha_{\mathrm{S}^{-}}$ CN (A, B, C, D, E, F, G, H, and I), 4 protein variants of $\alpha_{\mathrm{S}^{2}} \mathrm{CN}(\mathrm{A}, \mathrm{B}, \mathrm{C}$, and $\mathrm{D}), 12$ protein variants of $\beta-\mathrm{CN}\left(\mathrm{A}^{1}, \mathrm{~A}^{2}, \mathrm{~A}^{3}, \mathrm{~B}, \mathrm{C}, \mathrm{D}, \mathrm{E}, \mathrm{F}, \mathrm{G}, \mathrm{H}^{1}, \mathrm{H}^{2}\right.$, and I), and 13 protein variants $\left(A, B, B^{2}, C, D, E, F^{1}, F^{2}, G^{1}, G^{2}\right.$, $\mathrm{H}$, I, and $\mathrm{J}$ ) and 1 synonymous variant $\left(\mathrm{A}^{\mathrm{I}}\right)$ of $\kappa-\mathrm{CN}$ are known (Caroli et al., 2009). The majority of these casein variants are caused by SNP within the open reading frame resulting in amino acid exchanges in the mature protein (missense mutations). Other variants, such as $C S N 1 S 1^{*} A$ and $C S N 1 S 2^{*} D$, show deletions of several amino acids due to nucleotide exchanges that affect splice sites and hence are generated by exon skipping (Bouniol et al., 1993; Mohr et al., 1994).

Up to now, new variants were predominantly identified at the protein level by electrophoretic or isoelectrophoretic techniques. This allows the identification of variants differing in their electric charge, their molecular weight, or their isoelectric point (Caroli et al., 2009). At the DNA level, PCR-single-strand conformation polymorphism (PCR-SSCP) analysis is a common tool to detect and genotype casein variants (Prinzenberg et al., 1999). However, the identification of mutations in fragments larger than $200 \mathrm{bp}$ is difficult (Jaeckel et al., 1998). 
Table 1. Animals used in the sequencing of casein genes $(\mathrm{n}=356)$

\begin{tabular}{llll}
\hline Breed & Acronym & Origin & $\mathrm{n}^{1}$ \\
\hline Bos taurus $(\mathrm{n}=285)$ & & & \\
Angler & AN & Germany & 20 \\
Eringer & ER & Switzerland & 13 \\
German Red Pied & GR & Germany & 14 \\
German Yellow & GY & Germany & 25 \\
Highland Cattle & HL & United Kingdom & 27 \\
Hinterwälder & HW & Germany & 21 \\
Hungarian Grey Steppe & HG & Hungary & 20 \\
Jersey & JE & United Kingdom & 20 \\
Limpurger & LP & Germany & 25 \\
Pinzgauer & PG & Germany & 17 \\
Retinta & RE & Spain & 20 \\
Sarabi & SA & Iran & 22 \\
Shorthorn & SH & Germany & 16 \\
Vorderwälder & VW & Germany & 25 \\
Bos indicus $(\mathrm{n}=71)$ & & & 25 \\
Gir & GI & Brazil & 22 \\
Golpayegani & GO & Iran & 24 \\
Sistani & SI & Iran & 3. \\
\hline
\end{tabular}

${ }^{1}$ The number of successfully sequenced animals differed between the genes; details are given in Table 3 .

Over the past years, sequencing has become a feasible alternative to PCR-SSCP as costs have been reduced significantly. Nevertheless, this technique has often only been used for characterization of already known variants at the protein level (Ibeagha-Awemu et al., 2007; Lühken et al., 2009) or DNA level (Jann et al., 2002).

Furthermore, studies dealing with the identification of new casein variants often considered only European taurine cattle breeds, even if different variants were described and characterized in Bos indicus (Mahé et al., 1999; Ceriotti et al., 2003; Chen et al., 2008; Lühken et al., 2009).

The aim of this study was the identification of new variants of the 4 caseins $\alpha_{\mathrm{S}^{-}} \mathrm{CN}, \alpha_{\mathrm{S}^{2}} \mathrm{CN}, \beta-\mathrm{CN}$, and $\kappa-\mathrm{CN}$ at the DNA level. Therefore, we sequenced the open reading frame of the respective genes CSN1S1, CSN1S2, CSN2, and CSN3 in samples from both taurine and indicine cattle breeds. Furthermore, allele frequencies were calculated and analyzed for all examined breeds.

\section{MATERIALS AND METHODS}

\section{Animals and Samples}

The casein genes of 356 unrelated individuals of 17 breeds from 7 countries spanning 3 continents were analyzed in this study. Thirteen of the 17 investigated breeds belonged to the European Bos taurus cattle species and 3 to the Indian Bos indicus group. The native Iranian breed Sarabi has been assigned to the Indian Bos taurus group (Nassiry et al., 2008). The breeds are summarized in Table 1. The DNA was extracted either from full blood or semen samples, applying a modified protocol according to Miller et al. (1988). The semen samples were subjected to an additional treatment with dithiothreitol to break the disulfide bonds of nucleoprotamines.

\section{DNA Sequencing}

For sequence analysis, the genomic GenBank (http://www.ncbi.nlm.nih.gov/genbank) sequence NC_007304.4 of bovine CSN1S1, CSN2, CSN1S2, and CSN3 3 and the PRIMER 3 software (Rozen and Skaletsky, 2000) were used to generate primer pairs for PCR amplification. Primer pairs were created only for exons that represent the open reading frame of each gene. Each primer pair spanned up to 3 exons and the $5^{\prime}$ and $3^{\prime}$ flanking intronic sequences, resulting in a total of 42 PCR products with length from 434 to $888 \mathrm{bp}$ (Supplemental Table 1, available online at http://www. journalofdairyscience.org/). The PCR amplifications with their respective primer pairs were performed in a $12-\mu \mathrm{L}$ reaction volume including $20 \mathrm{ng}$ of genomic DNA, $0.2 \mu M$ concentration of each primer, $200 \mu M$ deoxyribonucleotide triphosphate (dNTP), and $5 \mathrm{U}$ of Taq DNA polymerase (Invitek GmbH, Berlin, Germany) in the reaction buffer supplied by the manufacturer. Cycling conditions using a MJ Research PTC-200 thermal cycler (Global Medical Instrumentation Inc., Ramsey, $\mathrm{MN})$ were as follows: initial denaturation at $94^{\circ} \mathrm{C}$ for $5 \mathrm{~min}, 35$ cycles each of $94^{\circ} \mathrm{C}$ for $30 \mathrm{~s}, 62^{\circ} \mathrm{C}$ for $60 \mathrm{~s}$, $72^{\circ} \mathrm{C}$ for $90 \mathrm{~s}$, and a final extension step at $72^{\circ} \mathrm{C}$ for 10 min. After purification of the PCR products with thermosensitive alkaline phosphatase (FastAP; Fer- 
mentas GmbH, St. Leon-Rot, Germany) and exonuclease I (Fermentas $\mathrm{GmbH}$ ), sequencing was carried out with one of the correspondent primers using an $\mathrm{ABI}$ $3130 x l$ Genetic Analyzer and BigDye Terminator Cycle Sequencing Ready Reaction Kit (Applied Biosystems Inc., Foster City, CA). The obtained sequences were analyzed and compared with the genomic GenBank sequence NC_007304.4 using the software Sequencher 4.9 (Gene Codes Corp., Ann Arbor, MI). Allele frequencies for all observed variants of the 4 casein-encoding genes CSN1S1, CSN2, CSN1S2, and CSN3 were calculated by direct counting for all 17 examined breeds.

\section{Multidimensional Scaling}

To enhance the assignment of the breeds under consideration, especially the native Iranian breeds, to either the taurine or the indicine group, a nonmetric multidimensional scaling (nmMDS) approach (Kruskal, 1964) based on genome-wide SNP marker data was adopted. Therefore, marker data of 368 animals from 20 breeds (Table 2) were obtained from another project. There was an overlap of 192 animals with the sample used for sequencing of the casein genes. For some breeds, SNP data were available for all sequenced animals (Table 2). The animals were genotyped for the Illumina SNP50v1 BeadChip (Illumina Inc., San Diego, CA) comprising a total of 54,001 markers. Less than $2 \%$ genotypes were missing per individual. After excluding X-chromosomal SNP and unmapped markers according to the bovine genome assembly UMD3.1, a total of 43,049 autosomal markers remained. An additional 115 markers were excluded due to a minor allele frequency below $1 \%$ or more than $10 \%$ missing genotypes across all genotyped animals, resulting in 42,934 markers remaining for the analysis. From these data, an identical by state (IBS) distance matrix was calculated as 1 - IBS using PLINK software (Purcell et al., 2007), which was then subjected to nmMDS. Although metric MDS has been proposed by Purcell et al. (2007) to assess population structure, the advantages of nmMDS have been pointed out by Zhu and $\mathrm{Yu}$ (2009). Scaling was done within the $\mathrm{R}$ statistical environment ( $\mathrm{R}$ Foundation for Statistical Computing, Vienna, Austria) using the function isoMDS from the library MASS (http://www.stats. ox.ac.uk/pub/MASS4), which implements nmMDS according to Kruskal (1964). To determine the optimal dimensionality, scaling was carried out with an increasing number of dimensions. For each final configuration, stress-1 according to Kruskal (1964) was determined. This was repeated until stress for the final configuration was below a threshold of 0.05 , which was achieved for $\mathrm{k}=8$. The first 2 dimensions obtained from this
Table 2. Animals used for multidimensional scaling (MDS) ${ }^{1}$

\begin{tabular}{lcc}
\hline Breed & $\begin{array}{c}\text { Genotyped } \\
\text { animals for } \\
\text { MDS (no.) }\end{array}$ & $\begin{array}{c}\text { Overlap with } \\
\text { resequencing } \\
\text { sample (no.) }\end{array}$ \\
\hline Angler & 21 & - \\
Charolais & 15 & - \\
Eringer & 25 & - \\
German Holstein & 25 & - \\
German Red Pied & 2 & 25 \\
German Yellow & 25 & 22 \\
Gir & 22 & 22 \\
Golpayegani & 22 & 6 \\
Highland Cattle & 9 & 21 \\
Hinterwälder & 21 & - \\
Istrian Cattle & 18 & 25 \\
Jersey & 23 & - \\
Limpurger & 26 & 22 \\
Pinzgauer & 9 & 24 \\
Romagnola & 25 & - \\
Sarabi & 23 & 25 \\
Sistani & 25 & 192 \\
Shorthorn & 4 & - \\
Vorderwälder & 1 & - \\
Zebu & 368 & \\
Sum & &
\end{tabular}

${ }^{1}$ Given are the numbers of animals genotyped for the Illumina Bovine SNP50 BeadChip (Illumina Inc., San Diego, CA; total and by breed) and the fraction of those animals overlapping with the sample used for resequencing the casein genes.

solution were plotted against each other (Figure 1) and visually inspected to assess population structure.

\section{RESULTS}

\section{Sequencing of Casein Genes}

The open reading frames of the 4 casein genes were sequenced in 356 animals belonging to 14 taurine and 3 indicine breeds. The numbers of animals successfully sequenced differs between the genes; details are given below and in Table 2 . The analysis revealed 4 variants of the $\alpha_{S 1}$-CN-encoding gene CSN1S1, 10 variants of the $\beta$-CN-encoding gene CSN2, 4 variants of the $\alpha_{\mathrm{S} 2}$-CN-encoding gene CSN1S2, and 5 variants of the $\kappa$-CN-encoding gene CSN3. Thus, 23 DNA sequence variants were identified, of which 5 with a predicted effect on the protein sequence were previously unknown. However, they were identified on the genomic DNA (gDNA) level and the effect on the mature protein was merely predicted and presumed the expression of these variants. Before names are finally assigned to the variants, their existence should be validated on a protein level. Nevertheless, we suggested preliminary names for the newly identified variants according to the existing alphabetical order to improve readability when referring to the new variants. The counted allele frequencies of all identified variants are summarized in Table 3. 


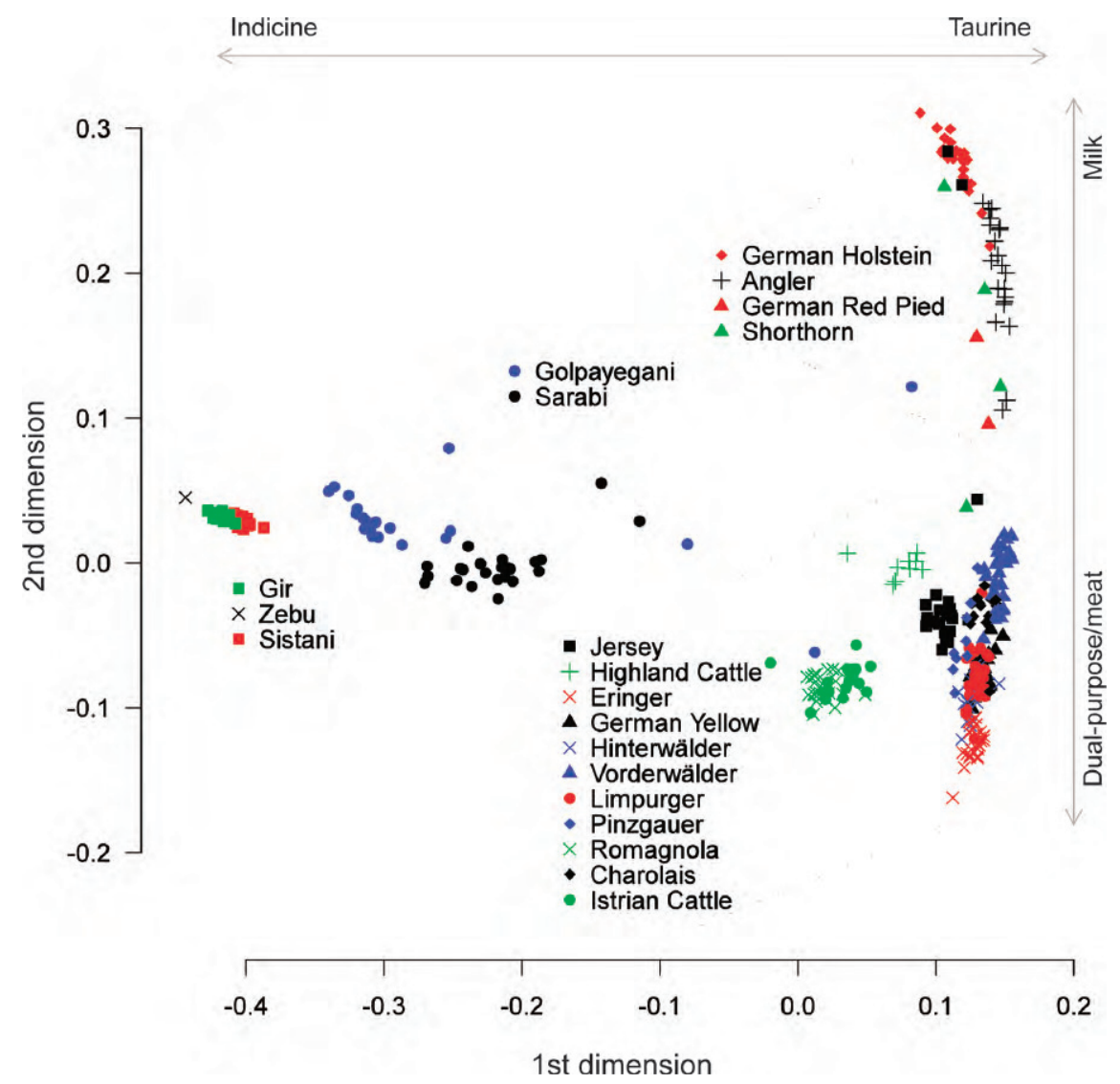

Figure 1. Graphical representation of the multidimensional scaling (MDS) analysis. The first 2 dimensions are plotted against each other. The first dimension clearly differentiates between taurine and indicine breeds.

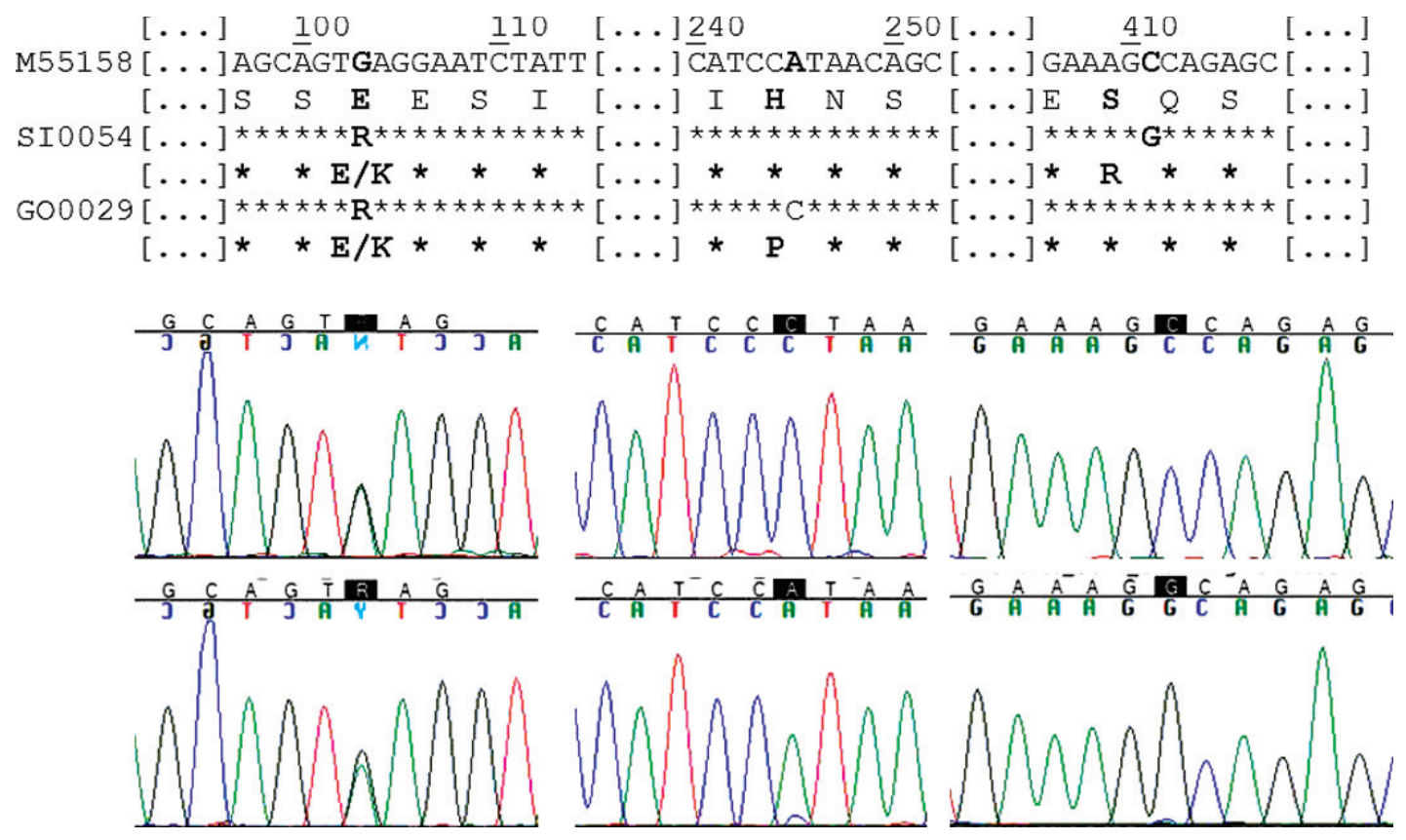

Figure 2. Comparison of CSN2 coding sequence positions 103, 245, and 411 and the deduced amino acid sequence of GenBank (http://www. ncbi.nlm.nih.gov/genbank) sequence M55158 (representing variant $C S N 2{ }^{*} A^{1}$ ), with both animals showing the c.103 G > A nucleotide exchange leading to the new variant $C S N 2^{*} J$. 
Table 3. Number of examined animals per breed (n) and counted allele frequencies at the 4 casein-encoding genes CSN1S1, CSN2, CSN1S2, and CSN3

\begin{tabular}{|c|c|c|c|c|c|c|c|c|c|c|c|c|c|c|c|c|c|c|c|c|c|c|c|c|c|c|c|}
\hline \multirow[b]{2}{*}{ Breed $^{1}$} & \multicolumn{5}{|c|}{$C S N 1 S 1$} & \multicolumn{11}{|c|}{ CSN2 } & \multicolumn{5}{|c|}{ CSN1S2 } & \multicolumn{6}{|c|}{ CSN3 } \\
\hline & $\mathrm{n}$ & B & $\mathrm{C}$ & I & J & $\mathrm{n}$ & $\mathrm{A}^{1}$ & $\mathrm{~A}^{2}$ & $\mathrm{~A}^{3}$ & B & $\mathrm{C}$ & $\mathrm{F}$ & I & J & K & $\mathrm{L}$ & $\mathrm{n}$ & A & B & $\mathrm{D}$ & $\mathrm{E}$ & $\mathrm{n}$ & A & $\mathrm{A}^{\mathrm{I}}$ & B & $\mathrm{E}$ & $\mathrm{H}$ \\
\hline \multicolumn{28}{|c|}{ Bos taurus } \\
\hline AN & 19 & 1.00 & $-^{2}$ & - & - & 16 & 0.44 & 0.28 & - & 0.06 & - & 0.22 & - & - & - & - & 19 & 0.95 & - & 0.05 & - & 20 & 0.55 & - & 0.43 & 0.03 & - \\
\hline ER & 13 & 0.77 & 0.23 & - & - & 13 & 0.12 & 0.54 & - & 0.27 & - & - & 0.08 & - & - & - & 13 & 1.00 & - & - & - & 13 & 0.50 & - & 0.50 & - & - \\
\hline GR & 14 & 0.93 & 0.07 & - & - & 12 & 0.42 & 0.21 & 0.08 & 0.08 & - & 0.21 & - & - & - & - & 14 & 1.00 & - & - & - & 14 & 0.71 & - & 0.29 & - & - \\
\hline GY & 24 & 1.00 & - & - & - & 23 & 0.30 & 0.52 & - & 0.02 & 0.11 & 0.02 & 0.02 & - & - & - & 23 & 0.93 & - & 0.07 & - & 20 & 0.45 & - & 0.55 & - & - \\
\hline HL & 26 & 1.00 & - & - & - & 24 & 0.19 & 0.81 & - & - & - & - & - & - & - & - & 24 & 1.00 & - & - & - & 27 & 1.00 & - & - & - & - \\
\hline HW & 20 & 0.93 & 0.08 & - & - & 21 & 0.21 & 0.79 & - & - & - & - & - & - & - & - & 21 & 0.98 & - & 0.02 & - & 20 & 0.75 & - & 0.25 & - & - \\
\hline $\mathrm{HG}$ & 20 & 0.73 & 0.28 & - & - & 15 & 0.30 & 0.67 & - & 0.03 & - & - & - & - & - & - & 20 & 0.93 & 0.05 & 0.03 & - & 19 & 0.55 & 0.05 & 0.34 & - & 0.05 \\
\hline JE & 20 & 0.68 & 0.33 & - & - & 19 & 0.05 & 0.63 & - & 0.26 & - & - & 0.05 & - & - & - & 20 & 1.00 & - & - & - & 19 & 0.42 & - & 0.58 & - & - \\
\hline LP & 22 & 1.00 & - & - & - & 22 & 0.07 & 0.77 & - & 0.11 & 0.02 & - & 0.02 & - & - & - & 20 & 0.98 & - & 0.03 & - & 23 & 0.80 & - & 0.20 & - & - \\
\hline $\mathrm{PG}$ & 17 & 0.74 & 0.26 & - & - & 10 & 0.25 & 0.65 & - & 0.05 & 0.05 & - & - & - & - & - & 17 & 0.74 & 0.26 & - & - & 17 & 0.59 & - & 0.18 & - & 0.24 \\
\hline $\mathrm{RE}$ & 19 & 0.32 & 0.68 & - & - & 19 & 0.11 & 0.89 & - & - & - & - & - & - & - & - & 20 & 1.00 & - & - & - & 20 & 0.13 & - & 0.88 & - & - \\
\hline SA & 20 & 0.53 & 0.40 & 0.03 & 0.05 & 22 & 0.14 & 0.75 & - & 0.11 & - & - & - & - & - & - & 22 & 0.89 & 0.09 & - & 0.02 & 22 & 0.30 & 0.02 & 0.34 & - & 0.34 \\
\hline $\mathrm{SH}$ & 15 & 1.00 & - & - & - & 14 & 0.43 & 0.57 & - & - & - & - & - & - & - & - & 15 & 1.00 & - & - & - & 15 & 0.60 & - & 0.40 & - & - \\
\hline VW & 23 & 0.87 & 0.13 & - & - & 19 & 0.11 & 0.50 & - & 0.39 & - & - & - & - & - & - & 24 & 0.90 & - & 0.10 & - & 23 & 0.78 & - & 0.20 & 0.02 & - \\
\hline \multicolumn{28}{|c|}{ Bos indicus } \\
\hline GI & 7 & 0.14 & 0.50 & 0.21 & 0.14 & 25 & - & 0.74 & - & 0.08 & - & - & - & - & 0.16 & 0.02 & 7 & 0.71 & 0.29 & - & - & 7 & 0.07 & 0.36 & 0.07 & - & 0.50 \\
\hline GO & 20 & 0.48 & 0.48 & 0.05 & - & 21 & 0.07 & 0.71 & - & 0.14 & 0.05 & - & - & 0.02 & - & - & 20 & 0.88 & 0.13 & - & - & 21 & 0.31 & 0.02 & 0.40 & - & 0.26 \\
\hline SI & 21 & 0.07 & 0.88 & 0.05 & - & 24 & - & 0.90 & - & 0.02 & - & - & 0.06 & 0.02 & - & - & 22 & 0.64 & 0.36 & - & - & 23 & 0.28 & 0.13 & 0.09 & - & 0.50 \\
\hline
\end{tabular}

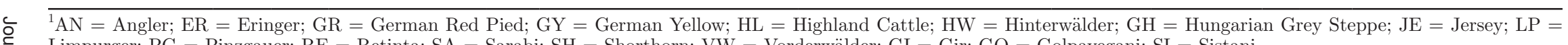
Limpurger; $\mathrm{PG}=$ Pinzgauer; $\mathrm{RE}=$ Retinta; $\mathrm{SA}=$ Sarabi; $\mathrm{SH}=$ Shorthorn; VW = Vorderwälder; GI = Gir; GO = Golpayegani; $\mathrm{SI}=$ Sistani.

Dashes indicate that the correspondent variant was not present in the breed. 
Table 4. Protein variants detected at the $C S N 1 S 1$ locus $^{1}$

\begin{tabular}{|c|c|c|c|c|c|}
\hline Protein variant & $\begin{array}{l}\text { Polymorphism compared } \\
\text { with genomic reference }\end{array}$ & $\begin{array}{l}\text { Location } \\
\text { within gene }\end{array}$ & $\begin{array}{l}\text { Amino acid } \\
\text { exchange }\end{array}$ & \multicolumn{2}{|c|}{ No. of breeds } \\
\hline CSN1S1*B & None (reference) & - & - & 14 & 3 \\
\hline CSN1S1*I & c. $296 \mathrm{~A}>\mathrm{T}^{2}$ & Exon 11 & Glu99Asp & 1 & 3 \\
\hline CSN1S1*J & c. $543 G>T^{2}$ & Exon 17 & Val182Phe & 1 & 1 \\
\hline
\end{tabular}

${ }^{1}$ Alterations of the coding sequence are described in comparison with the cDNA of the genomic reference sequence X59856.2 representing the preprotein variant $C S N 1 S 1^{*} B$. The protein variant newly identified within the current study is highlighted in boldface. The letter assigned to the variant is proposed within the current study.

${ }^{2}$ In addition to c. $619 \mathrm{~A}>\mathrm{G}$.

\section{CSN1S1}

The open reading frame of $C S N 1 S 1$ was completely sequenced in 320 animals. Three nonsynonymous nucleotide substitutions were identified by comparing with a genomic reference sequence representing variant $C S N 1 S 1^{*} B$ (accession no. X59856.2; Table 4). One of these polymorphisms was previously unknown. The known nucleotide exchange c.619A $>\mathrm{G}$ distinguishes variant $C S N 1 S 1^{*} C$ from $C S N 1 S 1{ }^{*} B$, which was identified in all examined breeds. Actually, in 5 of the 14 taurine breeds (Angler, German Yellow, Highland Cattle, Limpurger, and Shorthorn) a fixation for the B allele was observed (Table 3). The variant $C S N 1 S 1{ }^{*} C$ was present in all other breeds. The nucleotide substitution c. $296 \mathrm{~A}>\mathrm{T}$ characterizes variant $C S N 1 S 1^{*} I$. All animals carrying this substitution also showed the c.619A > G nucleotide exchange. Variant CSN1S1*I was identified in all Iranian breeds and Gir. A third nucleotide substitution c.543G $>\mathrm{T}$ in exon 17 leading to the amino acid exchange p.Val182Phe was identified in the breeds Sarabi and Gir. This polymorphism and the predicted amino acid exchange have not been described before. According to the alphabetical order of casein variants, we suggest to preliminarily name it $C S N 1 S 1^{*} J$.

\section{CSN2}

The CSN2 gene was sequenced in a total of 319 animals. Gene CSN2 showed the highest genetic variability among the casein genes within our sample. Ten nonsynonymous nucleotide substitutions were identified within the open reading frame by comparing with a genomic reference sequence representing variant CSN2 $^{*} A^{1}$ (accession no. M55158; Table 5). Three out of the identified polymorphisms have not been described previously. Animals carrying variant $\operatorname{CSN}^{*} A^{1}$ were observed in all taurine breeds, but only in 1 indicine breed (Golpayegani). The nucleotide substitution c.245A > $\mathrm{C}$ designates variant $C S N^{2}{ }^{*} A^{2}$. It was identified in all examined breeds and represented the most frequent variant except for the red cattle breeds Angler and German Red Pied. The variant $\operatorname{CSN}^{*} A^{3}$ is defined by the nucleotide exchange c.363C $>\mathrm{A}$ in addition to c.245A > C and was present only in German Red Pied

Table 5. Protein variants detected at the CSN2 locus based on resequencing of genomic DNA ${ }^{1}$

\begin{tabular}{|c|c|c|c|c|c|}
\hline Protein variant & $\begin{array}{l}\text { Polymorphism as compared } \\
\text { with genomic reference }\end{array}$ & $\begin{array}{l}\text { Location } \\
\text { within gene }\end{array}$ & $\begin{array}{l}\text { Amino acid } \\
\text { exchange }\end{array}$ & \multicolumn{2}{|c|}{ No. of breeds } \\
\hline $\mathrm{CSN}^{*} \mathrm{~A}^{1}$ & None (reference) & - & - & 14 & 1 \\
\hline $\mathrm{CSN}^{*} \mathrm{~A}^{3}$ & c. $363 \mathrm{C}>\mathrm{A}^{2}$ & Exon 7 & His121Gln & 1 & - \\
\hline $\mathrm{CSN}_{2}{ }^{*} \mathrm{~B}$ & c. $411 \mathrm{C}>\mathrm{G}$ & Exon 7 & Ser137Arg & 10 & 3 \\
\hline $\mathrm{CSN} 2 * \mathrm{C}$ & c. $154 \mathrm{G}>\mathrm{A}^{3}$ & Exon 6 & Glu52Lys & 3 & 1 \\
\hline CSN2*J & c. $103 \mathrm{G}>\mathrm{A}$ & Exon 4 & Glu35Lys & - & 2 \\
\hline CSN2*K & c. $580 \mathrm{C}>\mathrm{G}^{2}$ & Exon 7 & Pro194Ala & - & 1 \\
\hline $\mathrm{CSN}^{2} * \mathrm{~L}$ & c. $635 \mathrm{~T}>\mathrm{C}$ & Exon 7 & Val212Ala & - & 1 \\
\hline
\end{tabular}

${ }^{1}$ Alterations of the coding sequence are described in comparison with the cDNA of the genomic reference sequence M55158 representing the preprotein variant $C S 2^{*} A 1$. The protein variants newly identified within the current study are highlighted in boldface. The letters assigned to these variants are proposed within the current study.

${ }^{2}$ In addition to c. $245 \mathrm{~A}>\mathrm{C}$.

${ }^{3}$ Together with a dephosphorylation of the 50th amino acid (serine). 
cattle. Similarly, the variant $C S N 2{ }^{*} I$ carries the nucleotide substitution c.322A $>\mathrm{C}$ in exon 7 along with the c.245A $>\mathrm{C}$ mutation. It was present in 4 taurine (Eringer, German Yellow, Jersey, and Limpurger) and in 1 indicine breed (Sistani). Another nucleotide substitution in exon 7 characterizes variant $C S N 2{ }^{*} B$, which was observed in all indicine and in the majority of the taurine breeds. The amino acid exchange p.Pro167Leu caused by the nucleotide substitution c.500C $>$ T determines variant $C S N 2{ }^{*} F$, which was observed only in taurine breeds (Angler, German Yellow, and German Red Pied). Finally, the already known variant $C S N 2{ }^{*} C$ was identified in 3 taurine breeds, namely German Yellow, Pinzgauer, and Limpurger and the indicine breed Golpayegani. This variant differs from variant $C S N 2^{*} A^{1}$ by the nucleotide substitution c. $154 \mathrm{G}>\mathrm{A}$ in exon 6 leading to the amino acid exchange p.Glu52Lys. Moreover, variant $C S N 2^{*} C$ is characterized by a dephosphorylation of the 50th amino acid residue serine, but this was not further investigated in our study.

In addition to these 7 known nucleotide substitutions, 3 nonsynonymous variants were discovered that have not been described before. The nucleotide substitution c.103G > A in exon 4 leads to the amino acid exchange p.Glu35Lys. According to the alphabetical order of casein variants, we suggest $C S N 2^{*} J$ as a preliminary name. This variant was present in 2 animals belonging to the indicine breeds Golpayegani and Sistani, respectively. A further previously unknown polymorphism was identified in exon 7 . The c. $580 \mathrm{C}>\mathrm{G}$ nucleotide exchange leads to the amino acid exchange p.Pro194Ala and we propose to name this variant $C S N 2{ }^{*} K$. This variant was only observed in 6 animals belonging to the indicine breed Gir. All sequenced animals homozygous for the nucleotide substitution c.580C $>\mathrm{G}$ were also homozygous for the nucleotide substitution c.245A > $\mathrm{C}$ defining variant $C S{ }^{2} 2^{*} A^{2}$. The new nucleotide substitution c.635T $>\mathrm{C}$ likewise occurring in exon 7 leads to the amino acid exchange p.Val212Ala. This variant, which we refer to as $C S N 2^{*} L$, was discovered only in 1 animal of the indicine breed Gir.

\section{CSN1S2}

Successful sequencing in 321 animals revealed 3 nonsynonymous nucleotide substitutions within the open reading frame of the CSN1S2 gene as compared with GenBank sequence M94327 representing variant CSN1S2*A (Table 6). Animals without any nucleotide substitution and, hence, carrying variant $C S N 1 S 2^{*} A$ were observed in all examined breeds. Six of the 14 analyzed taurine breeds showed a fixation of this variant. The nucleotide substitution c.68C $>\mathrm{T}$ in exon 3 leads to the amino acid exchange p.Ser23Phe and distinguishes variant $C S N 1 S 2^{*} A$ from $C S N 1 S 2 * B$, which was observed in the Iranian breeds and Gir as well as Pinzgauer and Hungarian Grey Steppe. A further already known variant is $C S N 1 S 2^{*} D$. It is caused by the nucleotide exchange c.221G $>\mathrm{T}$ leading to the skipping of exon 8 and, hence, resulting in the deletion of the amino acid residues 51 to 59. Six taurine (Angler, German Yellow, Hinterwälder, Limpurger, Hungarian Grey Steppe, and Vorderwälder), but no indicine breed showed this allele.

A new polymorphism exon 3 was observed in one Sarabi animal. The c.64G > A nucleotide substitution leads to the amino acid exchange p.Val22Ile in the protein. According to the alphabetical order of already described variants we propose to call this new variant CSN1S2*E

\section{CSN3}

Sequencing of the open reading frame of CSN3 was successful in a total of 323 animals. It revealed 4 nonsynonymous and 1 synonymous nucleotide substitutions by comparing with GenBank sequence AY380228 corresponding to variant $C S N 3^{*} A$ (Table 7). All identified polymorphisms reside within exon 4 and represent already published variants. Animals without any nucleotide substitution and, hence, carrying variant $C S N 3^{*} A$ were present in all examined breeds. The synonymous nucleotide exchange c.513A $>$ G determining

Table 6. Protein variants detected at the CSN1S2 locus based on resequencing of genomic DNA ${ }^{1}$

\begin{tabular}{|c|c|c|c|c|c|}
\hline $\mathrm{CSN} 1 \mathrm{~S} 2 * \mathrm{~B}$ & c. $68 \mathrm{C}>\mathrm{T}$ & Exon 3 & Ser23Phe & 3 & 3 \\
\hline CSN1S2*D & c. $221 \mathrm{G}>\mathrm{T}$ & Exon 8 & Glu74Asp $^{2}$ & 6 & - \\
\hline CSN1S2*E & c. $64 \mathrm{G}>\mathrm{A}$ & Exon 3 & Val22Ile & 1 & - \\
\hline
\end{tabular}

${ }^{1}$ Alterations of the coding sequence are described in comparison with the cDNA of the genomic reference sequence M94327 representing the preprotein variant $\operatorname{CSN}_{S} 2^{*} A$. The protein variant newly identified within the current study is highlighted in boldface. The letters assigned to these variants are proposed within the current study.

${ }^{2}$ Leads to the skipping of exon 8 and, hence, to the deletion of the amino acids 51 to 59 . 
Table 7. Protein variants detected at the CSN3 locus based on sequencing of genomic DNA ${ }^{1}$

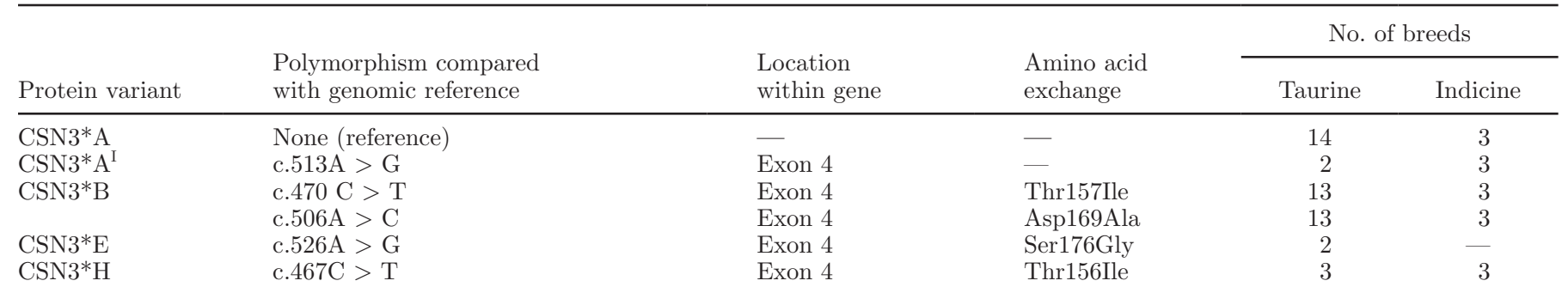

${ }^{1}$ Alterations of the coding sequence are described in comparison with the cDNA of the genomic reference sequence AY380228 representing the preprotein variant $C S N 3^{*} A$.

variant $C S N 3^{*} A^{I}$ was identified in the Iranian breeds, Gir, and Hungarian Grey Steppe. Two nonsynonymous nucleotide substitutions c. $470 \mathrm{C}>\mathrm{T}$ and c.506A $>$ $\mathrm{C}$ lead to the amino acid exchanges p.Thr157Ile and p.Asp169Ala, respectively. These 2 polymorphisms correspond to variant $\operatorname{CSN}^{*} B$ and were present in all analyzed breeds except for Highland cattle.

The amino acid exchange p.Ser176Gly caused by the nucleotide substitution c.526A $>\mathrm{G}$ determines variant $C S N 3^{*} E$ and was only observed in 2 taurine breeds (Angler and Vorderwälder). The last variant of the $\kappa$-CN-encoding gene present in our sample was $\operatorname{CSN}^{*} H$, characterized by the amino acid exchange p.Thr156Ile. It was observed in the Iranian breeds, Gir, Hungarian Grey Steppe, and Pinzgauer.

\section{Multidimensional Scaling}

The results of the MDS are summarized in Figure 1. The first dimension clearly differentiated between indicine and taurine cattle with the Zebu breeds and Sistani as one extreme and various European taurine breeds as the other. The Iranian breeds Sarabi and Golpayegani clustered together halfway between Zebus and taurine animals, with the Golpayegani closer to the indicine breeds.

The second dimension differentiated among the taurine breeds. The one extreme was formed by milk breeds such as German Holstein and Angler, whereas the Southern German dual-purpose breeds clustered together with Swiss Eringer and meat breeds at the other extreme. One exception was Jersey cattle, which mainly fell into the latter cluster except for 3 outliers, which were probably crossbred with Holstein. The Jersey animals formed a distinct subcluster close to Highland cattle, thus representing British breeds. This did not apply to the Shorthorn animals that could be found in the cluster of milk breeds. This is plausible, because the analyzed animals belonged to a type of the breed specially selected for milk performance and thus showing various degrees of introgression from other milk breeds. The Angler animals represented a further exception, as they were very dispersed along the second axis, which probably reflects various degrees of Holstein introgression. Finally, the Podolian breeds Romagnola and Istrian cattle formed a distinct cluster closer to the indicine breeds. These findings were, however, out of the scope of this study and were not regarded further.

\section{DISCUSSION}

\section{Sequencing of Casein Genes}

The method of direct sequencing was proven as an excellent approach for the detection of milk protein variants within the current study. It allowed us to identify not only known but also 5 new casein variants. The great advantage of sequencing over the electrophoretic and isoelectrophoretic techniques (i.e., isoelectric focusing) is the possibility of detecting all the variants carried out by the sequenced animals, including new variants and not only those represented by amino acid exchanges altering the electric charge of the protein. Within this study, only 1 out of 5 newly identified variants showed an amino acid exchange that considerably altered the electric charge of the protein. In variant CSN2* J, the amino acid $\mathrm{Glu}_{35}$ is exchanged to the amino acid $\mathrm{Lys}_{35}$ and, hence, the isoelectric point of the 35th amino acid changes from 3.22 to 9.74 , from acid to basic. As technological advances have led to a considerable reduction in sequencing time and costs, this method has become affordable and feasible even for small working groups.

The disadvantage exists, however, that nothing can be said about the expression of new protein variants detected by sequencing of genomic DNA or possible splice variants. Although one previously identified splice site mutation was detected in this study $\left(C S N 1 S 2{ }^{*} D\right.$; Table 6 ), the sequencing of mRNA would have been desirable to detect new splice variants. This was, however, not possible, because no appropriate milk or tissue samples suitable for RNA extraction or protein purification 
were available for most of the breeds. Furthermore, variants due to posttranslational mechanisms such as the dephosphorylation of serine, which is known for $C S N 2{ }^{*} C$, cannot be detected by sequencing of genomic DNA. Reviewing the protein variants identified to date, it becomes, however, obvious that most of them can be identified based on the underlying variation on a genomic DNA level. To confirm the novelty of the identified variants, a BLAST search (http://blast.ncbi. nlm.nih.gov/Blast.cgi) of the generated sequence data against public databases was conducted. One CSN2 sequence (accession no. EF628290) originating from Bos taurus was found containing the nucleotide substitution coding for $\mathrm{Ala}_{212}$. However, no related publication is available and no name was proposed for this variant.

The counted allele frequencies presented in Table 3 are predominantly in agreement with previous studies (Formaggioni et al., 1999; Jann et al., 2004; IbeaghaAwemu et al., 2007; Caroli et al., 2010), although the number of analyzed individuals per breed was comparably small within the current study.

\section{Taurine Versus Indicine Casein Variants}

Three of the breeds analyzed in the current study, namely Gir, Sistani, and Golpayegani, have previously been classified as indicine breeds. The Sarabi breed, which along with Sistani and Golpayegani represents a native Iranian cattle breed, has on the other hand been assigned to the Bos taurus group (Nassiry et al., 2008). In accordance with Lühken et al. (2009), we identified variant $C S N 1 S 1^{*} I$ in all Bos indicus breeds as well as in the Sarabi breed. Similar observations were made for the variants $C S N 1 S 1^{*} J, C S N 1 S 2^{*} B, C S N 3^{*} A^{I}$, and $C S N 3^{*} H$, which are also considered to be Bos indicus specific. All of these variants were present in our Sarabi sample. In contrast, typical taurine variants $\left(C S N 2{ }^{*} C\right.$, $C S N 2^{*} F, C S N 2^{*} I, C S N 1 S 2^{*} D$, and $C S N 3^{*} E$ ) were not observed in the Sarabi sample. Nassiry et al. (2008) estimated genetic differentiation and relationships between the 4 Iranian cattle breeds Sarabi, Sistani, Golpayegani and Najdi (Bos indicus). They found evidence for genetic exchange between these 4 populations, especially between Sarabi and Golpayegani due to their geographic adjacency. Sistani originates from the Sistan and Baluchestan provinces close to the Pakistani border (southeast), where indicine breeds have initially been domesticated. The Sarabi breed bears the name of the city Sarab located in the Azerbaijan province (northwest) 2,500 km away and Golpayegani represents the central region of Iran.

The findings are in agreement with the results of the MDS analysis (Figure 1). The first dimension clearly differentiates between taurine and indicine breeds. Sarabi and Golpayegani are located halfway between the 2 extreme groups, indicating an admixture of the 2 cattle species in these breeds. This is further supported by the fact that the typical taurine variant $C S N 2{ }^{*} C$ occurs with a frequency of 0.07 within the Golpayegani breed. Notably, the degree of crossbreeding seems to correspond with the geographic distribution of the Iranian breeds.

Further Bos taurus breeds showing typical Bos indicus casein variants were the Pinzgauer $\left(C S N 1 S 2^{*} B\right.$ and $C S N 3^{*} H$ ) and the Hungarian Grey Steppe cattle $\left(C S N 1 S 2^{*} B, C S N 3^{*} A^{I}\right.$ and $\left.C S N 3^{*} H\right)$. For the latter breed no SNP chip data were available. However, samples of Romagnola and Istrian cattle were included, which along with Hungarian Grey Steppe belong to the Podolian cattle breeds. These animals formed a tight cluster close to but clearly distinct from the other European breeds. A similar differentiation has already been reported (Negrini et al., 2007). The occurrence of Bos indicus-specific variants within this group of southern or eastern European breeds has previously been shown. Jann et al. (2004) identified the variants $\operatorname{CSN}^{*}{ }^{*}{ }^{1}$ and $\mathrm{CSN}^{*} H$ in the Anatolian Black and Turkish Grey Steppe and suggested Bos indicus introgression as the cause of these observations. Counted allele frequencies of 0.05 of $C S N 1 S 2^{*} B, C S N 3^{*} A^{I}$, and $C S N 3^{*} H$, respectively, of the Hungarian Grey Steppe cattle indicate a Bos indicus introgression in this breed as well.

The identification of variants $C S N 1 S 2^{*} B$ and $\mathrm{CSN}^{*} H$ at relatively high frequency of 0.27 and 0.23 , respectively, in the Pinzgauer cattle is an interesting addition to the findings of Caroli et al. (2010). They identified $C S N 1 S 2{ }^{*} B$ by isoelectric focusing in the Pinzgauer cattle at a frequency of 0.202 and also observed $C S N 3^{*} H$ in a subsample of 59 resequenced animals. Together, these results indicate an introgression of Bos indicus not only in the Hungarian Grey Steppe cattle, but also in the Pinzgauer.

All newly identified casein variants were exclusively present in indicine breeds, including Sarabi. Thus, we propose to classify them as Bos indicus-specific variants. Due to the fact that the Gir, although sampled in Brazil, are descendants from animals originally imported from India in the 1930s and 1940s, the new variants should more precisely be termed as Indian Bos indicus specific.

\section{Relevance of Newly Identified Variants}

The relatively high $C S N 1 S 1^{*} J$ frequency of 0.14 in the Gir breed and the additional occurrence in Sarabi predispose this variant for the use in future casein ge- 
netic diversity studies, especially in Bos indicus breeds. The same should be applied for variant $C S N 2{ }^{*} K$, which occurred with a frequency of 0.16 in the Gir cattle.

The new variant $C S N 1 S 2^{*} E$ as well as the new variant $C S N 2{ }^{*} L$ were only found in heterozygous condition in 1 Sarabi and 1 Gir animal, respectively. Nevertheless, both variants seem to be quite interesting and should not be disregarded. The nucleotide substitution c. $64 \mathrm{G}>\mathrm{A}$ determining $C S N 1 S 2^{*} E$ is known in the goat to determine variant $C S N 1 S 2^{*} F$ leading to the same amino acid exchange p.Val22Ile (Ramunno et al., 2001). The variant $C S N 2^{*} J$ was identified in heterozygous condition in only 2 animals. These animals belonged, however, to different indicine breeds, namely Sistani and Golpayegani, indicating a variant that is not restricted to a single breed.

The fact that variant $C S N 2^{*} L$, which was identified in an indicine breed here, has previously been found in a Bos taurus animal (GenBank sequence EF628290) indicates the occurrence of this variant, though probably just in low frequencies, in Bos indicus as well as in Bos taurus breeds.

\section{Evolution of the Newly Identified Casein Variants}

Considering the evolutionary pathway of the newly identified casein variants, it can be presumed that the variant $C S N{ }^{*} K$ might have evolved from variant CSN2*A'. All animals homozygous for the nucleotide substitution c.580C > G were also homozygous for the nucleotide substitution c.245A $>\mathrm{C}$ defining variant $C_{S N 2^{*}} A^{2}$. Similarly, the new variant $C S N 1 S 1^{*} J$ seems to have evolved from variant $C S N 1 S 1^{*} C$, as the Sarabi cattle homozygous for c.543G $>\mathrm{T}$ also carried the substitution defining $C S N 1 S 1^{*} C$. This also applies to the c.296A $>$ T substitution defining $C S N 1 S 1^{*} I$, as this variant also originated from variant $C S N 1 S 1^{*} C$ (Lühken et al., 2009).

The Sarabi cattle showing the nucleotide substitution c. $64 \mathrm{G}>\mathrm{A}$ in heterozygous condition determining the new variant $C S N 1 S 2^{*} E$ also showed the nucleotide substitution c.68C $>\mathrm{T}$ in heterozygous condition. However, because the linkage phases were unknown, the evolution of $C S N 1 S 2{ }^{*} E$ could not definitely be determined. The new variant $C S N 2^{*} L$ seems to have evolved from the reference variant $C S N^{2} A^{2}$. The Gir cattle showing the $C_{S N 2}{ }^{*} L$ determining nucleotide substitution c.635T > $\mathrm{C}$ showed no other nucleotide substitution.

The reconstruction of the evolution of variant $\mathrm{CSN2}^{*} J$ proved to be even more difficult. The underlying nucleotide substitution c.103G > A was present in heterozygous state in 2 animals belonging to the breeds Golpayegani and Sistani, respectively. Whereas the Golpayegani cattle also carried the nucleotide substitu- tion belonging to variant $C S N 2{ }^{*} B($ c. $411 \mathrm{C}>\mathrm{G})$ in homozygous form, the Sistani cattle additionally showed the substitution causing variant $C S N 2 * A^{2}$ (c.245A > C) in homozygous form (Figure 2). These special findings lead to 3 different explanation approaches. Because both animals carrying the newly identified nucleotide exchange belonged to different breeds, it might be possible that the substitution occurred independently within the 2 breeds. A more likely explanation is the occurrence of a recombination in 1 of the 2 animals. Thus, variant $C S N^{2}{ }^{*} J$ evolved either from $C S N 2^{*} A^{2}$ or $C S N^{2}{ }^{*} B$. Finally, it is possible that $C S N^{2}{ }^{*} J$ is an ancestral allele and both variants $C S N 2^{*} A^{2}$ and $C S N 2{ }^{*} B$ evolved from it.

\section{CONCLUSIONS}

In the current study, additional genetic variation of bovine casein genes, especially in Bos indicus breeds, was discovered by direct sequencing. The identification of those variants is of interest not only for phylogenetic studies and evolution studies on milk protein genes, but also for the effect of milk protein variants on milk composition. However, because no milk or RNA samples of the herein-analyzed animals were available, future studies should address the expression of the 5 new variants on protein level to verify the findings.

\section{ACKNOWLEDGMENTS}

This project was funded by the German Federal Ministry of Education and Research (Bonn, Germany) within the competence network "Food Chain Plus" (FoCus, grant no. 0315539A).

\section{REFERENCES}

Boettcher, P. J., A. Caroli, A. Stella, S. Chessa, E. Budelli, F. Canavesi, S. Ghiroldi, and G. Pagnacco. 2004. Effects of casein haplotypes on milk production traits in Italian Holstein and Brown Swiss cattle. J. Dairy Sci. 87:4311-4317.

Bouniol, C., C. Printz, and J.-C. Mercier. 1993. Bovine $\mathrm{a}_{\mathrm{s} 2}$-casein D is generated by exon VIII skipping. Gene 128:289-293.

Caroli, A., R. Rizzi, G. Lühken, and G. Erhardt. 2010. Short communication: Milk protein genetic variation and casein haplotype structure in the Original Pinzgauer cattle. J. Dairy Sci. 93:12601265.

Caroli, A. M., S. Chessa, and G. J. Erhardt. 2009. Invited review: Milk protein polymorphisms in cattle: Effect on animal breeding and human nutrition. J. Dairy Sci. 92:5335-5352.

Ceriotti, G., A. Caroli, R. Rizzi, and C. Crimella. 2003. Genetic relationships among taurine (Bos taurus) and zebu (Bos indicus) populations as revealed by blood groups and blood proteins. J. Anim. Breed. Genet. 120:57-67.

Chen, S. Y., V. Costa, M. Azevedo, M. Baig, N. Malmakov, G. Luikart, G. Erhardt, and A. Beja-Pereira. 2008. Short communication: New alleles of the bovine $\kappa$-casein gene revealed by resequencing and haplotype inference analysis. J. Dairy Sci. 91:3682-3686. 
Farrell, H. M., Jr., R. Jimenez-Flores, G. T. Bleck, E. M. Brown, J. E. Butler, L. K. Creamer, C. L. Hicks, C. M. Hollar, K. F. Ng-KwaiHang, and H. E. Swaisgood. 2004. Nomenclature of the proteins of cows' milk—Sixth revision. J. Dairy Sci. 87:1641-1674.

Formaggioni, P., A. Summer, M. Malacarne, and P. Mariani. 1999. Milk protein polymorphism: Detection and diffusion of the genetic variants in Bos genus. Ann. Fac. Med. Vet. Univ. Parma 19:127-165.

Hayes, H. C., and E. J. Petit. 1993. Mapping of the $\beta$-lactoglobulin gene and of an immunoglobulin $M$ heavy chain-like sequence to homoeologous cattle, sheep, and goat chromosomes. Mamm. Genome 4:207-210.

Ibeagha-Awemu, E. M., E.-M. Prinzenberg, O. C. Jann, G. Lühken, A. E. Ibeagha, X. Zhao, and G. Erhardt. 2007. Molecular characterization of bovine $C S N 1 S 2^{*} B$ and extensive distribution of Zebu-specific milk protein alleles in European cattle. J. Dairy Sci. 90:3522-3529.

Jaeckel, S., J. T. Epplen, M. Kauth, B. Miterski, F. Tschentscher, and C. Epplen. 1998. Polymerase chain reaction-single strand conformation polymorphism or how to detect reliably and efficiently each sequence variation in many samples and many genes. Electrophoresis 19:3055-3061.

Jann, O., G. Ceriotti, A. Caroli, and G. Erhardt. 2002. A new variant in exon VII of bovine $\beta$-casein gene (CSN2) and its distribution among European cattle breeds. J. Anim. Breed. Genet. 119:65-68.

Jann, O. C., E. M. Ibeagha-Awemu, C. Özbeyaz, P. Zaragoza, J. L. Williams, P. Ajmone-Marsan, J. A. Lenstra, K. Moazami-Goudarzi, and G. Erhardt. 2004. Geographic distribution of haplotype diversity at the bovine casein locus. Genet. Sel. Evol. 36:243-257.

Kruskal, J. B. 1964. Multidimensional-scaling by optimizing goodness of fit to a nonmetric hypothesis. Psychometrika 29:1-27.

Lühken, G., A. Caroli, E. M. Ibeagha-Awemu, and G. Erhardt. 2009. Characterization and genetic analysis of bovine $\mathrm{a}_{\mathrm{s} 1}$-casein I variant. Anim. Genet. 40:479-485.

Mahé, M.-F., G. Miranda, R. Queval, A. Bado, P. S. Zafindrajaona, and F. Grosclaude. 1999. Genetic polymorphism of milk proteins in African Bos taurus and Bos indicus populations. Characterization of variants $\alpha_{\mathrm{S} 1}-\mathrm{Cn} H$ and $\kappa$-Cn J. Genet. Sel. Evol. 31:239-253.

Miller, S. A., D. D. Dykes, and H. F. Polesky. 1988. A simple salting out procedure for extracting DNA from human nucleated cells. Nucleic Acids Res. 16:1215.
Mohr, U., D. Koczan, D. Linder, G. Hobom, and G. Erhardt. 1994. A single point mutation results in A allele-specific exon skipping in the bovine $\alpha_{\mathrm{s} 1}$-casein mRNA. Gene 143:187-192.

Nassiry, M. R., F. E. Shahroudi, M. Tahmoorespur, and A. Javadmanesh. 2008. The diversity of BoLA-DRB3 gene in Iranian native cattle. Asian-australas. J. Anim. Sci. 21:465-470.

Negrini, R., I. J. Nijman, E. Milanesi, K. Moazami-Goudarzi, J. L. Williams, G. Erhardt, S. Dunner, C. Rodellar, A. Valentini, D. G. Bradley, I. Olsaker, J. Kantanen, P. Ajmone-Marsan, and J. A. Lenstra., and the European Cattle Genetic Diversity Consortium. 2007. Differentiation of European cattle by AFLP fingerprinting. Anim. Genet. 38:60-66.

Nilsen, H., H. G. Olsen, B. Hayes, E. Sehested, M. Svendsen, T. Nome, T. Meuwissen, and S. Lien. 2009. Casein haplotypes and their association with milk production traits in Norwegian Red cattle. Genet. Select. Evol. 41:24.

Prinzenberg, E. M., I. Krause, and G. Erhardt. 1999. SSCP analysis at the bovine CSN3 locus discriminates six alleles corresponding to known protein variants $(\mathrm{A}, \mathrm{B}, \mathrm{C}, \mathrm{E}, \mathrm{F}, \mathrm{G})$ and three new DNA polymorphisms $\left(\mathrm{H}, \mathrm{I}, \mathrm{A}^{1}\right)$. Anim. Biotechnol. 10:49-62.

Purcell, S., B. Neale, K. Todd-Brown, L. Thomas, M. A. R. Ferreira, D. Bender, J. Maller, P. Sklar, P. I. W. de Bakker, M. J. Daly, and P. C. Sham. 2007. PLINK: A tool set for whole-genome association and population-based linkage analyses. Am. J. Hum. Genet. $81: 559-575$.

Ramunno, L., G. Cosenza, M. Pappalardo, E. Longobardi, D. Gallo, N. Pastore, P. Di Gregorio, and A. Rando. 2001. Characterization of two new alleles at the goat CSN1S2 locus. Anim. Genet. $32: 264-268$.

Rozen, S., and H. Skaletsky. 2000. Primer3 on the WWW for general users and for biologist programmers. Methods Mol. Biol. 132:365-386.

Threadgill, D. W., and J. E. Womack. 1990. Genomic analysis of the major bovine milk protein genes. Nucleic Acids Res. 18:69356942.

Wedholm, A., L. B. Larsen, H. Lindmark-Månsson, A. H. Karlsson, and A. Andrén. 2006. Effect of protein composition on the cheesemaking properties of milk from individual dairy cows. J. Dairy Sci. 89:3296-3305.

Zhu, C., and J. Yu. 2009. Nonmetric multidimensional scaling corrects for population structure in association mapping with different sample types. Genetics 182:875-888. 